\title{
Novel Image-guided Surgery of Gallbladder Cancer by Indocyanine Green Fluorescence Navigation
}

\author{
Satoru Seo ${ }^{1}$, Rei Toda ${ }^{1}$, Hiroto Nishino ${ }^{1}$, Ken Fukumitsu ${ }^{1}$, Takamichi Ishii ${ }^{1}$, Kojiro Taura ${ }^{1}$, \\ Takashi Nitta $^{2}$, Toshimi Kaido ${ }^{1}$, Kyoichi Takaori ${ }^{1}$, Etsuro Hatano ${ }^{3}$, Shinji Uemoto ${ }^{1}$
}

'Department of Surgery, Graduate School of Medicine, Kyoto University, Kyoto, Japan 2Department of Surgery, Kobe City Medical Center West Hospital, Kobe, Japan ${ }^{3}$ Department of Surgery, Hyogo College of Medicine, Hyogo, Japan

\section{ABSTRACT}

Background: There has been a debate as to the extent of hepatic resection for T2 gallbladder cancer. While some surgeons advocate resection of the segments 4 a and 5 , gallbladder bed resection is also performed by others. A fluorescent imaging technique with selective injection of indocyanine green can provide real-time anatomic guidance within the operative field for hepatobiliary surgery. This study was performed to evaluate the usefulness of fluorescent navigation surgery for gallbladder cancer.

Methods: Four patients underwent fluorescent navigation surgery for gallbladder cancer. As a source of fluorescence, indocyanine green was injected into the cystic artery. The liver surface was observed with a photodynamic eye and the perfusion area of indocyanine green was resected en bloc. All patients underwent lymph node dissection. Results: The patients comprised two men and two women with a mean age of 72 years. The mean operation time was 439.8 minutes, the mean blood loss was $504.5 \mathrm{ml}$, and the mean postoperative hospital stay was 16.5 days. The pathological tumor depth was T1b in one patient, T2 in two, and $\mathrm{T} 3$ in one. Lymph node metastasis was present in one patient. A negative surgical margin was obtained in all patients. The disease recurred in lymph nodes in the nodepositive patient and the remaining three patients are alive with no evidence of disease to date. Conclusions: Navigation surgery utilizing ICG fluorescence angiography via the gallbladder artery may provide a clue to the optimal areas of en bloc hepatic resection for T2 gallbladder cancer.

Key words: fluorescence navigation surgery, gallbladder cancer, ICG

\section{INTRODUCTION}

The prognosis of gallbladder cancer remains bleak despite that aggressive surgical treatments have been attempted recently. Among gallbladder cancers, pathological T2 gallbladder cancer (invasion beyond the proper muscle layer but no serosal invasion) is considered the optimal candidates for radical surgical resection, given that the incidence of either hepatic metastasis or lymph node
Corresponding author:

Satoru Seo, MD, PhD

Department of Surgery

Graduate school of Medicine Kyoto

University

54 Kawahara-cho, Syogoin

Sakyo-ku, Kyoto 606-8507, Japan

Telephone number: 85-75-751-3234

Fax number: 85-75-751-4263

E-mail: rutosa@kuhp.kyoto-u.ac.jp 
metastasis of T3 gallbladder cancer is significantly higher. When managing clinical T2 gallbladder cancer, en bloc hepatectomy is valuable for ensuring an adequate tumor-free margin on the liver side, and prophylactic resection of hepatic parenchyma may help to prevent hepatic recurrence. In 1954, Glenn and Hays (1) first proposed a radical surgical procedure including gallbladder bed resection (en bloc resection of adjacent liver tissue of the gallbladder with $a \geq 1-\mathrm{cm}$ rim at the time of cholecystectomy) and lymphatic tissue dissection within the hepatoduodenal ligament.

It has been advocated that the most likely site of hepatic metastases in patients with advanced gallbladder carcinoma is the segment $4 a$ (S4a) and segment (S5) according to the Couinaud's nomenclature for anatomical segments of the liver (2-4). Therefore, S4a+S5 hepatic resection has been commonly performed procedure for $\mathrm{T} 2$ gallbladder cancer by some surgeons, while hepatic resection of the gallbladder bed resection also has been performed by others. According toa study of treatment for gallbladder cancer utilizing the Japanese Biliary Tract Cancer Registry, it was concluded that S4a+S5 hepatectomy was not superior to gallbladder bed resection (5). Besides, the mechanism of hepatic metastasis from gallbladder carcinoma is unknown and no consensus regarding the optimal extent of liver resection for $\mathrm{T} 2$ gallbladder cancer has been established.

A fluorescent imaging technique with indocyanine green (ICG) for navigation surgery has been introduced into clinical practice recently. The number of publications on fluorescent navigation surgery has dynamically increased since around the year 2000, indicating wide application of navigation surgery to many fields including hepatobiliary surgery. Aoki et al. (6) reported that the use of a near-infrared fluorescence imaging system is a novel and reliable intraoperative technique to identify hepatic segments and subsegments for anatomical hepatic resection.Kai et al. (7) reported that ICG fluorescence angiography is a useful method to visualize blood flow in hepatic parenchyma via the cholecystic vein in a real time manner, although they did not investigate cystic venous flow in patients with gallbladder carcinoma. They speculated that the dynamic blood flow of the gallbladder may change as the tumor progresses and causes the occlusion of some cholecystic veins (7). Furthermore, the area of perfusion in the liver from then gallbladder may vary from patients to patients. Therefore, it became important for us to investigate each patient with gallbladder carcinoma individually with the fluorescent imaging technique with ICG. The purpose of this study was to evaluate the usefulness of fluorescent navigation surgery for gallbladder cancer.

\section{MATERIAL AND METHOD}

\section{Study population}

From January 2012 to August 2016, four patients underwent fluorescent navigation surgery for clinical T2 gallbladder cancer at Kyoto University Hospital. The study protocol was approved by the ethics committee of the Graduate School of Medicine, Kyoto University (R1022). Three-dimensional images of the liver were created using SYNAPSE VINCENT software (Fujifilm Medical Co. Ltd., Tokyo, Japan) based on preoperative computed tomography images.

\section{Operation procedure}

Contrast-enhanced ultrasonography was routinely performed. First, the cystic artery was isolated, ligated, and cut. A 24-gauge angio-catheter was inserted into the cut end of the cystic artery and fixed by an arterial clip (fig. 1). As a source of fluorescence, ICG (Diagnogreen; Daiichi Sankyo Co., Tokyo, Japan) was injected into the cystic artery to determine the cutting line. The liver surface observed with a photodynamic eye (PDE) (8), the margin of area with fluorescence marked, and the area was resected en bloc. All patients underwent lymph node dissection in the hepatoduodenal ligament.

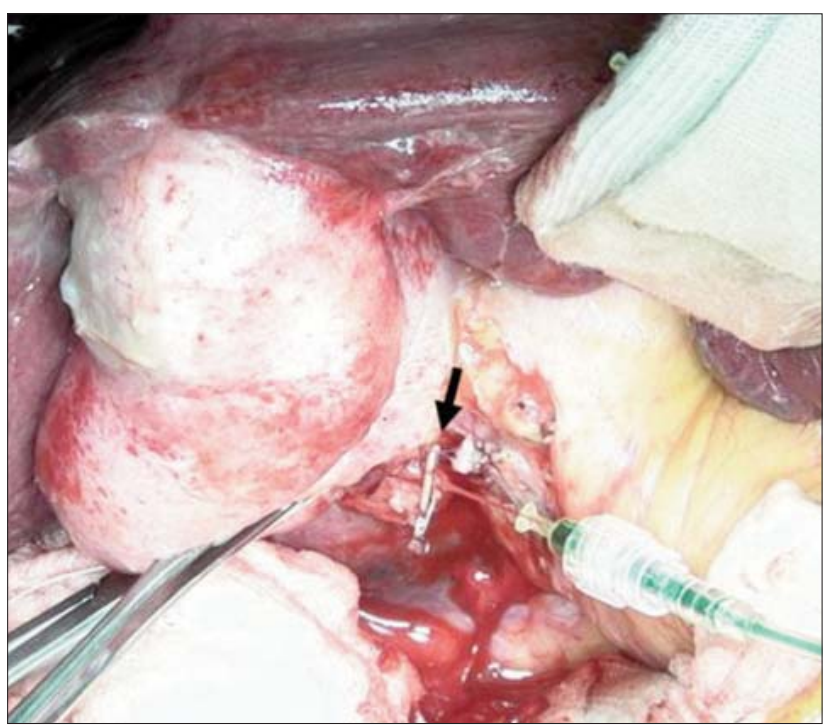

Figure 1 - A 24-gauge angio-catheter is inserted into the cut end of the cystic artery and fixed with an arterial clip 


\section{Fluorescence imaging system}

The PDE-Neo imaging system (Hamamatsu Photonics, Hamamatsu, Japan) detects ICG fluorescence and shows fluorescence images as demonstrated by the manufacturer's instructions. The light source, a lightemitting diode, emitted light at a wavelength of $760 \mathrm{~nm}$, and the detector is a charge-coupled device camera that subsequently filtered light with a wavelength of $820 \mathrm{~nm}$. Thirty-six light-emitting diodes are aligned on a board in an area, and the charge-coupled device camera is set in the center. The fluorescence signals are sent to a digital video processor for display on a television monitor. When ICG bounds to human plasma, the ICG evokes fluorescence which can be detected with the infrared observation camera in the PDE-Neo system.

\section{Histological examination}

The specimens were fixed in $10 \%$ formalin and embedded in paraffin. Serial $5-\mu \mathrm{m}$ sections were prepared for conventional light microscopic examination with hematoxylin-eosin staining analysis. Pathological diagnosis was made in accordance with the 8th UICC TNM Classification of Malignant Tumors (9).

\section{Surgical outcome}

The clinicopathological data, surgical outcomes, and survival data of all patients were extracted from the institutional database. The primary tumor characteristics and resection margins were confirmed based on the final histological finding. Major morbidity was defined as a grade $\geq 3$ complication according to the Clavien-Dindo classification (10). Patients were followed up with physical examination, evaluation of tumor marker levels (carcinoembryonic antigen, cancer antigen 19-9), and

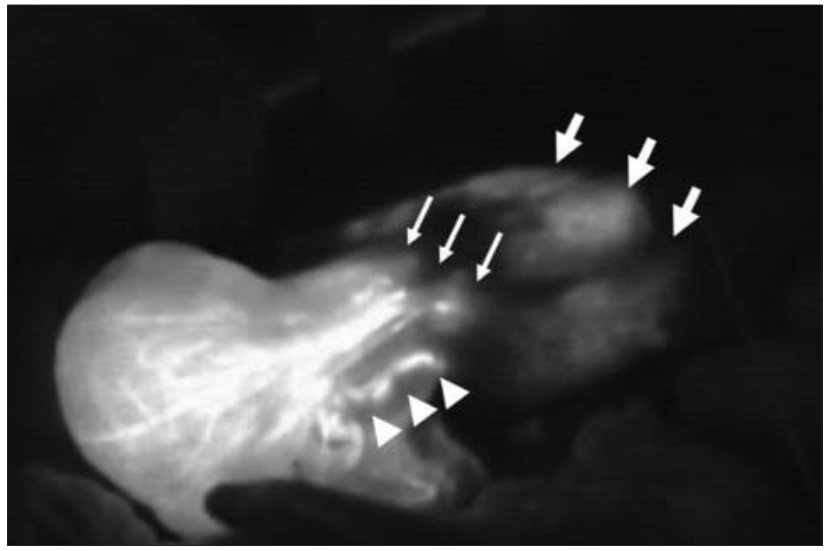

Figure 2 - Fluorescence of the cholecystic veins (arrow heads) and that of hepatic parenchyma perfused via the gallbladder bed route (thin arrows) and via the hepatic hilum route (thick arrows)

computed tomography of the chest, abdomen, and pelvis at least once every 3 months.

\section{RESULTS}

Mapping of the cystic venous area by fluorescence navigation

No adverse events were observed during this clinical study. The cholecystic veins were clearly visualized by the PDE after the injection of ICG into the cholecystic artery in all cases. As Kai et al. (7) has previously reported, the fluorescence of the cholecystic veins flows in two directions: one extended directly to the gallbladder bed, and the other extended to the hepatic hilum. In the present study also, both the fluorescence via gallbladder bed route and that of hepatic hilum route were visualized clearly and distinctively (fig. 2). None of the fluorescent mapping areas of the liver were consistent with the areas of $\mathrm{S} 4 \mathrm{a}+5$ resection orthose of gallbladder bed resection (fig. 3).
Figure 3 - The schema of the liver on the left side of each case shows the preoperative simulation of $\mathrm{S} 4 \mathrm{a}+5$ resection. None of the fluorescent mapping areas of the liver on the right side of each case were consistent with the areas of

$\$ 4 a+5$ resection or gallbladder bed resection
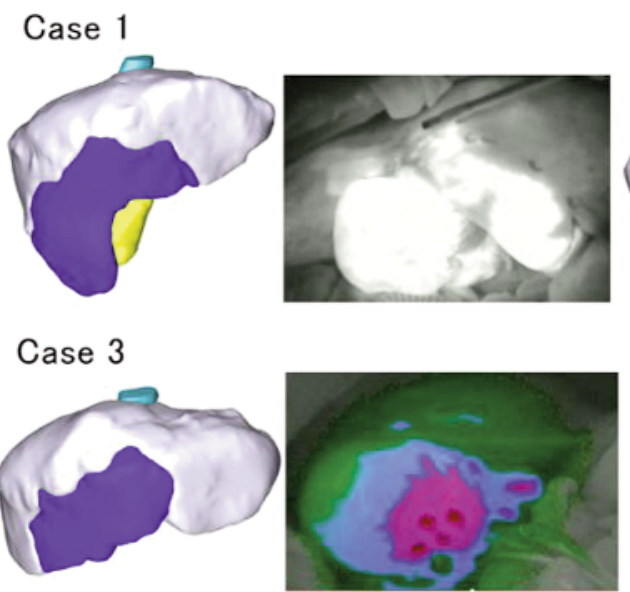

Case 2
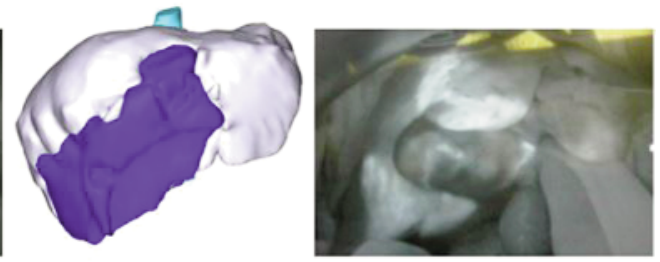

Case 4
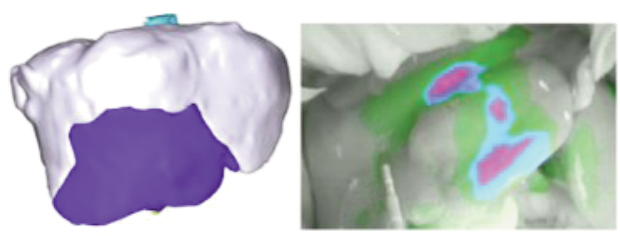


\section{Surgical outcomes}

The patient characteristics are summarized in table 1. The four patients comprised two men and two women with a median age of 72 years. The mean operation time was 439.8 minutes, the mean blood loss was $504.5 \mathrm{ml}$, and the mean postoperative hospital stay was 16.5 days. No postoperative complications occurred. The tumor depth according to the final pathological report was T1b in one patient, T2 in two, and T3 in one. Lymph node metastasis was present in one patient. A negative surgical margin was obtained in all patients. No metastasis was present in the resected liver.

The median follow-up duration for all patients was 27.8 months. No post-operative liver metastasis occurred in any patients so far. Lymph node metastasis was present in the resected specimen of one of the four patients. All patients were still alive at the present time.

\section{DISCUSSIONS}

The prognosis of patients with gallbladder cancer treated with simple cholecystectomy is poor and accordingly, there is a consensus that en bloc resection of the liver to a certain extent and regional lymph node excision is necessary for such patients (11-13). Ogura et al. (14) measured the distance between the gross border of the tumor and the resection plane, and their results suggested that a wide surgical margin was more likely to be achieved with $\mathrm{S} 4 \mathrm{a}+\mathrm{S} 5$ hepatic resection than with wedge resection. In the majority of Japanese institutions, gallbladder bed resection and $\mathrm{S} 4 \mathrm{a}+\mathrm{S} 5$ hepatectomy had been primarily practiced (15-21). A study of surgical treatment for gallbladder cancer utilizing the Japanese Biliary Tract Cancer Registry concluded that it was not clear whether or not S4a+S5 hepatectomy was superior to gallbladder bed resection for T2 gallbladder cancer (5). Accordingly, there is no definitive answer as to which of these two procedures is more useful.

Two important factors determine the optimal resec- tion margin as a means of prophylactic resection to prevent liver metastasis. Cholecystic venous flow was previously thought to be among the main metastatic routes of gallbladder cancer to the liver (22). The most preferable hepatic resection procedure to achieve microscopic RO resection is considered to be resection of the area into which the cholecystic veins flow. Yoshimitsu et al. (23) performed computed tomography angiography after superselective catheterization of the cholecystic artery and reported that the most frequent site of cholecystic venous drainage was S4 and S5 around the gallbladder. From these findings, we believe that the main route of cholecystic venous flow and drainage is S4 and S5. However, when analyzed in detail, the cystic veins that emerged from the body or fundus of the gallbladder mainly flowed into the subsegmental portal branches or sinusoids of S4a or S5. In contrast, those from the gallbladder neck flowed into the more proximal portal branches such as the umbilical portion, the right portal branches, and the anterior portal branches.In the present study, we found that that fluorescence existed not only in S4a and S5 but also in other segments. The anatomical pattern of interconnections of the vessels is not always the same as the pattern of blood flow in vivo; thus, intraoperative fluorescence angiography is necessary to identify the drainage area exactly. Endo et al. (24) reported that some patients with pathological T2 gallbladder carcinoma had microscopic liver metastases, which seemed to occur by a venous route. It has been considered that $\mathrm{pT} 2$ gallbladder cancer is the only type for which radical surgical resection may improve the survival. However, it is difficult to diagnose T2-stage gallbladder cancer preoperatively. In fact, two of the four patients in the present study did not have pT2 cancer but pT1 and pT3. The accuracy of preoperative diagnosis is also needed for the optimal treatment.

A fluorescent imaging technique in which ICG was recently introduced into hepatobiliary surgery providing anatomical descriptions and identifications within the operative field. Aoki et al. (6) reported that use of a near-infrared fluorescence imaging system is a novel

Table 1 - Patient characteristics

\begin{tabular}{|c|c|c|c|c|c|c|c|c|c|c|}
\hline & Gender & Age & $\begin{array}{l}\text { Operation } \\
\text { time }\end{array}$ & $\begin{array}{l}\text { Blood } \\
\text { loss }\end{array}$ & $\begin{array}{l}\text { Hospital } \\
\text { stay }\end{array}$ & pT stage & pN stage & $\begin{array}{c}\text { Adjuvant } \\
\text { chemotherapy }\end{array}$ & $\begin{array}{c}\text { Site of } \\
\text { recurrence }\end{array}$ & Prognosis \\
\hline 1 & female & 62 & 519 & 350 & 31 & T2 & N1 & GEM & lymph node & $5 y 5 m$ alive \\
\hline 2 & Male & 68 & 649 & 760 & 13 & T2 & NO & none & none & $2 \mathrm{y} 6 \mathrm{~m}$ alive \\
\hline 3 & male & 79 & 288 & 374 & 10 & T1b & NO & none & none & $1 \mathrm{y} 4 \mathrm{~m}$ alive \\
\hline 4 & female & 79 & 303 & 534 & 12 & T3 & NO & none & none & 1y alive \\
\hline
\end{tabular}


and reliable intraoperative technique to identify hepatic segments and subsegments for anatomical hepatic resection. ICG fluorescence imaging may serve as a reliable navigation tool especially in laparoscopic hepatectomy, in which gross inspection and palpation are limited (25).

ICG fluorescence angiography has the advantage of detecting the blood flow in a real time manner. However, there is a limitation of this procedure, which enables observation of the perfusion area only from the surface of the liver. Therefore, we are developing a new medical device, Medical Imaging Projection System (MIPS), to realize real-time navigation during hepatic resection in collaboration with Panasonic (Osaka, Japan)and reported the preliminary data (26). The MIPS projects of the fluorescence image directly to the liver continuously and enables surgical navigation to guide the dissection plane into the deep parts of the liver parenchyma throughout the procedure. The MIPS also prevents shifting of the visual focus, shaking of the surgeon's hands, and darkening of the operative field. We are planning to utilize the MIPS in the setting of gallbladder cancer in the near future.

\section{CONCLUSIONS}

In conclusion, navigation surgery utilizing ICG fluorescence angiography via the gallbladder artery may provide a clue to the optimal areas of en bloc hepatic resection for $\mathrm{T} 2$ gallbladder cancer.

\section{Disclosure of Interest}

Drs. Satoru Seo, Rei Toda, Hiroto Nishino, Ken Fukumitsu, Takamichi Ishii, Kojiro Taura, Takashi Nitta, Toshimi Kaido, Kyoichi Takaori, Etsuro Hatano, and Shinji Uemotohave no conflicts of interest or financial ties to disclose.

\section{REFERENCES}

1. Glenn F, Hays DM. The scope of radical surgery in the treatment of malignant tumors of the extrahepatic biliary tract. Surg Gynecol Obstet. 1954;99:529-41.

2. Shirai Y, Tsukada K, Ohtani T, Watanabe H, Hatakeyama K. Hepatic metastases from carcinoma of the gallbladder. Cancer. 1995;75: 2063-8.

3. Ohtsuka M, Miyazaki M, Itoh H, Nakagawa K, Ambiru S, Shimizu H, et al. Routes of hepatic metastasis of gallbladder carcinoma. Am J ClinPathol. 1998;109:62-8.

4. Kondo S, Nimura Y, Kamiya J, Nagino M, Kanai M, Uesaka K, et al. Mode of tumor spread and surgical strategy in gallbladder carcinoma. Langenbecks Arch Surg. 2002;387:222-8.

5. Horiguchi A, Miyakawa S, Ishihara S, Miyazaki M, Ohtsuka M,
Shimizu H, et al. Gallbladder bed resection or hepatectomy of segments $4 a$ and 5 for pT2 gallbladder carcinoma: analysis of Japanese registration cases by the study group for biliary surgery of the Japanese Society of Hepato-Biliary-Pancreatic Surgery $\mathrm{J}$ Hepatobiliary Pancreat Sci. 2013;20:518-24.

6. Aoki T, Yasuda D, Shimizu Y, Odaira M, Niiya T, Kusano T et al. Image-Guided Liver Mapping Using Fluorescence Navigation System with Indocyanine Green for Anatomical Hepatic Resection. World J Surg. 2008;32:1763-67.

7. Kai K, Satoh S, Watanabe T, Endo Y, Evaluation of cholecystic venous flow using indocyanine green fluorescence angiography $\mathrm{J}$ Hepatobiliary Pancreat Sci. 2010;17:147-51.

8. Ishizawa T, Saiura A, Kokudo N. Clinical application of indocyanine green-fluorescence imaging during hepatectomy. Hepatobiliary Surg Nutr. 2016;5(4):322-8.

9. International Union Against Cancer (UICC): TNM Classification of Malignant Tumors, ed 8. Wiley-Blackwell, 2017.

10. Dindo D, Demartines N, Clavien PA. Classification of surgical complications: a new proposal with evaluation in a cohort of 6336 patients and results of a survey. Ann Surg 2004; 240: 205-13.

11. Yamaguchi K, Chijiiwa K, Saiki S, Nishihara K, Takashima M, Kawakami K, et al. Retrospective analysis of 70 operations for gallbladder carcinoma. Br J Surg. 1997;84:200-4.

12. Foster JM, Hoshi H, Gibbs JF, lyer R, Javle $M$, Chu $Q$, et al. Gallbladder cancer: defining the indication for primary radical resection and radical re-resection. Ann SurgOncol. 2006;14:833-40.

13. Muratore A, Polastri R, Bouzari H, Vergara V, Capussotti L. Radical surgery for gallbladder cancer: a worthwhile operation? Eur J Oncol. 2000;26:160-3.

14. Ogura $Y$, Tabata M, Kawarada $Y$, Mizumoto R. Effect of hepatic invasion on the choice of hepatic resection for advanced carcinoma of the gallbladder: histologic analysis of 32 surgical cases. World $\mathrm{J}$ Surg. 1998;22:262-7.

15. Sikora S, Singh R. Surgical strategies in patients with gallbladder cancer: nihilism to optimism. J Surg Oncol. 2006;93:670-81.

16. Tsukada K, Hatakeyama K, Kurosaki I, Uchida K, Shirai Y, Muto T, et al. Outcome of radical surgery for carcinoma of the gallbladder according to the TNM stage. Surgery. 1996;120:816-21.

17. Kohya N, Miyazaki K. Hepatectomy of segment $4 a$ and 5 combined with extra-hepatic bile duct resection for T2 and T3 gallbladder carcinoma. J SurgOncol. 2008;97:498-502.

18. Kohya N, Kitahara K, Miyazaki K. Rational therapeutic strategy for T2 gallbladder carcinoma based on tumor spread. World Gastroenterol. 2010;16:3567-72.

19. Kai M, Chijiiwa K, Ohuchida J, Nagano M, Hiyoshi M, Kondo K. A curative resection improves the postoperative survival rate even in patients with advanced gallbladder carcinoma. J Gastrointest Surg. 2007;11:1025-32.

20. Suzuki S, Yokoi $Y$, Kurachi K, Inaba K, Ota S, Azuma M, et al. Appraisal of surgical treatment for pT2 gallbladder carcinomas. World J Surg. 2004:28:160-5.

21. Chijiiwa K, Nakano K, Ueda J, Noshiro H, Nagai E, Yamaguchi K, et al. Surgical treatment of patients with T2 gallbladder carcinoma invading the subserosal layer. J Am Coll Surg. 2001;192:600-7.

22. Yamanouchi H, Miyagawa K, Satoh S. Venous drainage of the gallbladder vein with reference to spread of carcinoma of the gallbladder (in Japanese). Tan to Sui. 1984:5:341-7.

23. Yoshimitsu K, Honda H, Kaneko K, Kuroiwa T, Irie H, Chijiiwa K, et al. Anatomy and clinical importance of cholecystic venous drainage: helical CT observations during injection of contrast medium into the cholecystic artery. AJR Am J Roentgenol. 1997;169:505-10.

24. Endo I, Shimada H, Takimoto A, Fujii Y, Miura Y, Sugita M et al. Microscopic liver metastasis: prognostic factor for patients with pT2 gallbladder carcinoma. World J Surg. 2004;7:692-696.

25. Terasawa M, Ishizawa T, Mise $\mathrm{Y}$, Inoue $\mathrm{Y}$, Ito $\mathrm{H}$, Takahashi $\mathrm{Y}$, Saiura A. Applications of fusion-fluorescence imaging using indocyanine green in laparoscopic hepatectomy. Surg Endosc. 2017;31(12): 5111-5118.

26. Nishino H, Hatano E, Seo S, Nitta T, Saito T, Nakamura M et al. Real-time Navigation for Liver Surgery Using Projection Mapping With Indocyanine Green Fluorescence: Development of the Novel Medical Imaging Projection System.Ann Surg. 2018 Jun; 267(6): 1134-1140. 